INVESTIGACIÓN/RESEARCH

\title{
LA VARIABLE DE GÉNERO EN LA MEDIDA DEL ESTRÉS Y EL BURNOUT EN LOS DOCENTES DE EDUCACIÓN INFANTIL Y PRIMARIA
}

Nuria Arís Redó1: Universidad Internacional de Cataluña. España nuria@cir.uic.es

\section{RESUMEN}

El presente artículo evalúa el modelo de medida del síndrome de Burnout mediante el instrumento de medición "Maslach Burnout Inventory" (MBI)2. Es un cuestionario de reconocido prestigio y muy utilizado para "medir" el síndrome de Burnout en los docentes. Se determinará el valor predictivo de algunos de los ítems de dicho cuestionario en relación a las respuestas según de la variable de género. La metodología utilizada fue transversal o de corte y la recogida de la información se efectuó a través de una encuesta. Se comprueba la existencia de diferencias significativas según la variable de género. Ciertamente estamos ante un importante problema que crece y tiene diferente caracterización en función del género. Pensamos que para futuros estudios y próximas investigaciones puede resultar interesante tener la referencia del valor predictivo ciertos ítems del MBI en una muestra de docentes españoles.

PALABRAS CLAVE: Profesores - Burnout - Medida - Género - MBI.

\footnotetext{
1 Autor correspondiente:

Nuria Arís Redó: Universidad Internacional de Cataluña. Barcelona. Vicedecana de la Facultad de Educación, España

Correo: nuria@cir.uic.es

2 De ahora en adelante usaremos las abreviación MBI para referiremos al instrumento: Maslach Burnout Inventory
} 


\title{
MEASUREMENT MODEL OF THE BURNOUT IN RELATION TO THE VARIABLE RESPONSES ACCORDING TO GENDER
}

\begin{abstract}
The purpose of this study was to assess the measurement model of the Burnout syndrome using the Maslach Burnout Inventory. It is a prestigious questionnaire and widely used to "measure" the Teacher Burnout syndrome._We want to determine the predictive value of some items of the questionnaire in relation to the variable responses according to gender. The used methodology was cross-sectional or of cut . The best fit of the data was achieved through a three-dimensional oblique solution (personal exhaustion, depersonalization and personal accomplishment). We think that for future studies and further research may be interesting to have the reference of the predictive value of certain items of the MBI in a sample of Spanish teachers.
\end{abstract}

KEY WORDS: Teachers - Burnout - measurement - gender - MBI.

\section{INTRODUCCIÓN}

Actualmente el rol docente ha cambiado $y$ se han constatado profundas modificaciones del contexto social y, concretamente, en las relaciones interpersonales que se establecen en el ámbito educativo. Basta echar una breve ojeada a los medios de comunicación, (televisión, prensa, etc.) para observar cómo se responsabiliza a los maestros de problemas de diversa índole, desde los que se dan tanto dentro del contexto escolar, en lo que atañe a las relaciones profesorado-alumnado (conflicto escolar, falta de asimilación de los contenidos curriculares ente los estudiantes, etc.), así como los referentes a cuestiones de tipo social, (violencia, desprestigio, adicciones, malos hábitos, etc.).

Podemos considerar que estrés laboral docente es un problema social y de salud pública. Algunos profesionales se ven forzados a implicarse durante muchas horas en las inquietudes de las personas con las que realizan su actividad laboral. Este es el caso de los maestros y docentes en general, cuya relación profesional se centra en el niño o el adolescente $y$, concretamente, en sus problemas, dificultades e inquietudes. El ejercicio continuado de la función docente en tales condiciones, va creando una acumulación de sensaciones, así como un desgaste personal y el profesorado se siente, personalmente $\mathrm{y}$ profesionalmente, abrumado $\mathrm{y}$ desconcertado, con fuertes contradicciones entre sus derechos y deberes. Todo ello puede conducir al estrés crónico y comportar el cansancio emocional y, finalmente, el estado definido como agotado, "quemado", o síndrome de Burnout.

Para conocer el origen de dicha problemática es preciso explicar que la definición de lo que vulgarmente es conocido como "estar quemado" - es reciente, apenas cuenta con unas pocas décadas. Este síndrome, fue descrito en 1974 por el psicólogo neoyorquino 
Herbert Freundenberger, que trabajaba con grupos de voluntariado que manifestaban un conjunto de síntomas físicos y psíquicos bien diferenciados: percepción de un estado de agotamiento, como consecuencia de trabajar intensamente, hasta el límite de no tomar en consideración las propias necesidades personales y "quemarse". Representa una perspectiva psicológica estrictamente individual, entendiendo que se trata de una respuesta a una interacción inadecuada entre profesionales excesivamente celosos de su trabajo y las necesidades y exigencias de los clientes.

La psicóloga social Cristina Maslach, prosiguió investigando al comienzo de la década de los 80 (Maslach, 1982) y estudió las respuestas emocionales de los empleados de profesiones de ayuda a personas eligiendo el mismo término "Burnout". Tal concepto fue definido por esta autora, como un síndrome de cansancio emocional que lleva a una pérdida de motivación y progresa hacia sentimientos de inadecuación y fracaso (Maslach y JAckson 1981). Considera que es el producto del estrés personal relacionado con una determinada condición laboral y característica de las profesiones de ayuda (maestros, educadores, enfermeras, etc.). La gran aportación de este nuevo enfoque supone abordar el problema del Burnout desde una perspectiva psicosocial (Calvete y Vila 2000).

Todo lo expuesto hasta este momento nos genera la motivación para llevar a cabo una investigación, para conocer cuál es la incidencia de esta problemática, considerando la incidencia según el género.

La actividad docente se caracteriza por realizarse en un ambiente en el que las relaciones personales, la negociación, la búsqueda de consenso son elementos que influyen y determinan la práctica pedagógica, el desarrollo de la profesionalidad docente, y la forma de desarrollar las prácticas educativas en un contexto social y político específico. Pero en el ámbito educativo, que históricamente se ha caracterizado por una alta presencia femenina nos surge la inquietud de investigar las diferencias en función de la variable de género. En esta ocasión nuestro interés lo centramos en la potencialidad del instrumento utilizado para medir el grado de afectación según la variable de género.

En el diseño de nuestro estudio utilizamos un instrumento de prestigio y solidez científica, reconocido por la sociedad NorteAmericana de Psicología, denominado Maslach Burnout Inventory que nos permitió determinar el grado de estrés y Burnout, en función de la intensidad con la que los docentes manifiestan unas determinadas sensaciones.

A pesar de todo, ciertamente un estudio doctoral ${ }^{3}$ sobre el estrés en el profesorado en activo, pone de manifiesto que los maestros se caracterizan por una percepción positiva de su propia competencia personal, así como por un alto componente

\footnotetext{
${ }^{3}$ Arís, N. (2008). El síndrome de Burnout en los docentes de eduación Infantil y educación primaria en la Zona del Valles Occidental. Barcelona: http://www.tdx.cat/TDX-1027108-171248
} 
vocacional. También manifiestan tener la sensación, muy generalizada, de haber dado el máximo de sí mismos. En este sentido queremos resaltar que esto nos indica el esfuerzo que este colectivo ya está realizando.

\section{METODOLOGÍA}

Concretamente nuestra hipótesis se sustenta en la premisa que en función del genero hay diferencias significativas en la vivencia y evolución del estrés docente y del síndrome de Burnout.

Para ello partimos de la consideración que ciertos ítems que configuran las tres subdimensiones del instrumento $\mathrm{MBI}^{4}$, podrían tener diferente impacto predictivo muy especialmente en relación a las respuestas en función de la variable de género.

Consultando otros estudios sobre la validez factorial de dicho instrumento obtuvimos mayor fundamentación para proceder a establecer esta nueva investigación (GilMonte, P. y Peiro, J. M., 1999 y 2000; Calvete, E. y Vila, I, 2000; Chermiss, C. ,1992; Álvarez, E. y Fernández, L. 1991a Y 1991b). Por todo lo explicado, el presente estudio, tiene el objetivo de aportar información adicional sobre el cuestionario MBI, en concreto establecer la influencia de determinados ítems en cada subescala considerando la variable de género.

\section{ANÁLISIS Y DISCUSIÓN}

\section{Sujetos}

La muestra está constituida por 89 sujetos, 75 mujeres y 14 hombres que representa un porcentaje de $84,3 \%$ y $15,7 \%$ respectivamente. En cuanto a dicha composición por sexo, coincide con los datos que nos suministró el "Departament d'Ensenyament" de la Generalitat de Catalunya, la cual confirma la tendencia histórica de este sector que se ha caracterizado por una intensa feminización de la función docente.

Ello puede explicarse desde tendencias culturales muy arraigadas en la sociedad (Abraham, A ,1984; Álvarez, M; Obiols, M., 2009), que atribuían en la población femenina el cuidado y la educación de los niños sobre todo en las etapas de ecuación infantil y primaria objeto de nuestro estudio.

\footnotetext{
${ }^{4}$ Es un cuestionario que consta de veintidós ítems repartidos en tres sub escalas: Cansancio emocional, despersonalización y realización personal.
} 
Tabla 1: Distribución de la muestra por género:

\begin{tabular}{|c|c|c|c|}
\hline & & Frecuencia & Porcentaje \\
\hline \multirow[t]{3}{*}{ Válidos } & hombre & 14 & 15,73 \\
\hline & mujer & 75 & 84,27 \\
\hline & Total & 89 & 100,00 \\
\hline
\end{tabular}

\section{Instrumento: El Maslach Burnout Inventory}

Cabe explicar que este instrumento lleva el nombre de su autora, la psicóloga social norteamericana Cristina Maslach. Ella investigó al comienzo de la década de los 80, y estudió las respuestas emocionales de los empleados de profesiones de ayuda a personas (maestros, enfermeras, etc.), eligiendo el término "Burnout" -quemado-, para explicar lo que les sucedía.

Tales investigaciones (Maslach y Jackson, 1981; Maslach, 1982) le llevaron a desarrollar un instrumento denominado Maslach Burnout Inventory, (Maslach y Jackson, 1986, en versión española Seisdedos, 1997).

Dicho instrumento ha tenido una gran implementación en multitud de estudios sobre el estrés, y el sindrome de Burnout. Se trata de un cuestionario que consta de veintidós ítems repartidos en tres subescalas, cada una de las cuales se identifica con las tres dimensiones establecidas por los citados autores, como definitorias del síndrome de Burnout:

$\boldsymbol{A}$ Cansancio emocional, (CE), que valoran la vivencia de estar exhausto a nivel emocional debido a las demandas del trabajo. Consta de nueve ítems:

- Me siento emocionalmente agotado por mi trabajo

- Me siento cansado al final de la jornada de trabajo

- Me siento fatigado cuando me levanto por la mañana y tengo que enfrentarme a otro día de trabajo

- Trabajar todo el día con personas es un esfuerzo

- Me siento quemado por mi trabajo

- Me siento frustrado en mi trabajo

- Creo que estoy trabajando demasiado-

- Trabajar directamente con personas me produce estrés

- Me siento acabado

Despersonalización, (DP), constituida por cinco ítems, que mide el grado de frialdad y distanciamiento relacional:

- Creo que trato a algunas personas como si fueran objetos personales

- Me he vuelto insensible con la gente desde que ejerzo esta profesión 
- Me preocupa el hecho de que este trabajo me este endureciendo emocionalmente

- No me preocupa realmente lo que ocurre a algunas personas a las que doy servicio

- Creo que las personas que trato me culpan de algunos de sus problemas

Realización personal, $(R P)$, con ocho ítems evalúa los sentimientos de eficacia, competencia y realización de objetivos personales.

- Fácilmente comprendo cómo se sienten las personas

- Trato muy eficazmente los problemas de las personas

- Creo que estoy influyendo positivamente con mi trabajo en la vida de los demás

- Me siento muy activo

- Fácilmente puedo crear una atmósfera relajada con las personas a las que doy servicio

- Me siento estimulado después de trabajar en contacto con personas

- He conseguido muchas cosas útiles en mi profesión

- En mi trabajo trato los problemas emocionales con mucha calma

Se presenta en un formato de respuesta tipo escala de Likert ${ }^{5}$, y agrupa en frecuencias la situación descrita en cada ítem.

La obtención de puntuaciones altas en la escala CE y DP, y bajas en RP se relacionan con la presencia del Burnout, pudiendo contrastar los datos con los valores de referencia que se suministran en el protocolo del diseño original del instrumento. Esta distribución no es homogénea en cuanto a la cantidad de ítems para cada subescala e implica que estén medidas en escalas diferentes, por ello las puntuaciones mínimas y máximas óptimas para cada una de ellas son: de 0 a 54 para C.E., de 0 a 30 para D.P., y 0 a 48 para RP.

\section{Análisis Estadístico}

Entendemos que es importante conocer el valor predictivo que los ítems del MBI aportan en su propia dimensión (Cansancio Emocional, Despersonalización, y Realización Personal) desde un punto de vista de sujeto según su género.

\footnotetext{
5 Escala que consta de siete grados, que abarca de -nunca- a -todos los días-, con cinco grados intermedios, que son: pocas veces al año, una vez al mes o menos, unas pocas veces al mes, una vez a la semana, pocas veces a la semana.
} 
Para tal fin se utilizó el coeficiente de Cronbach', puesto que permite medir la consistencia interna de todos los ítems, globalmente y individualmente. Es decir, puntuar con un porcentaje elevado en un determinado ítem, puede ser interpretado como una clara señal de alerta, si dicho ítem tiene un valor predictivo potente dentro de la subescala. Todo ello considerando la posibilidad de diferencias relevantes entre hombres y mujeres.

En cada una de los siguientes apartados quedan explicitados los ítems que componen la subescala en cuestión y el resultado de cada ítem en referencia a la muestra total y también el resultado por separado en función de la variable 'sexo'.

2.1 Subescala Cansancio Emocional.

2.2 Subescala Despersonalización.

2.3 Subescala Realización personal.

\subsection{Subescala Cansancio Emocional:}

Tabla 2: Valores obtenidos globalmente y por grupos en C. E.:

\begin{tabular}{|l|l|l|l|l|}
\hline COD. ITEM & TOTAL & Hombre & Mujer \\
\hline B01 & $\begin{array}{l}\text { Me siento emocionalmente agotado por mi } \\
\text { trabajo }\end{array}$ & $67,71 \%$ & $51,64 \%$ & $69,80 \%$ \\
\hline B2 & $\begin{array}{l}\text { Me siento cansado al final de la jornada } \\
\text { de trabajo }\end{array}$ & $70,57 \%$ & $29,92 \%$ & $73,54 \%$ \\
\hline $\begin{array}{l}\text { Me siento fatigado cuando me levanto por } \\
\text { la mañana y tengo que enfrentarme a otro } \\
\text { dia de trabajo }\end{array}$ & $68,85 \%$ & $42,10 \%$ & $71,21 \%$ \\
\hline B03 & $\begin{array}{l}\text { Trabajar todo el dia con personas es un } \\
\text { esfuerzo }\end{array}$ & $76,44 \%$ & $67,85 \%$ & $76,94 \%$ \\
\hline B06 & Me siento quemado por mi trabajo & $66,42 \%$ & $35,12 \%$ & $69,14 \%$ \\
\hline B13 & Me siento frustrado en mi trabajo & $70,40 \%$ & $52,31 \%$ & $72,38 \%$ \\
\hline B14 & Creo que estoy trabajando demasiado & $72,67 \%$ & $37,91 \%$ & $74,49 \%$ \\
\hline B16 & Trabajar directamente con personas me $68,62 \%$ & $43,35 \%$ & $71,03 \%$ \\
\hline
\end{tabular}

\footnotetext{
6 Coeficiente alfa de Cronbach. Fórmula algorítmica que permite medir la fiabilidad real de un instrumento, tanto la global del cuestionario como la de cada ítem por separado. La fiabilidad es el aspecto de la cualidad de los registros observacionales que verifica si hay coincidencia entre los valores cuantitativos emitidos por el observador en dos momentos diferentes, o por dos observadores en el mismo momento temporal, (Arnau, 1996).
} 


\begin{tabular}{l|l|l|l|l|}
\multicolumn{2}{c|}{ produce estrés } & & & \\
\hline B20 & Me siento acabado & $72,20 \%$ & $51,96 \%$ & $70,40 \%$ \\
\hline
\end{tabular}

El ítem que más peso tiene en la puntuación de manera global en la subescala Cansancio Emocional, es "Trabajar todo el día con personas es un esfuerzo".

Observando las diferencias de respuesta en función de la variable género comprobamos que hay una única tendencia. Es decir, tanto hombres como mujeres coinciden en conferir a esta afirmación la máxima significación de su subescala, por lo que podemos considerar que dicho ítem tiene una mayor capacidad predicativa. Únicamente queremos destacar en el caso de las mujeres, que éstas lo manifiestan ligeramente más elevado que el porcentaje total. Observando el resultado en el ítem de menor porcentaje, si detectamos una variación significativa entre de los resultados totales y por grupos de hombre / mujer.

En la muestra global, el ítem de menor porcentaje es "Me siento frustrado en mi trabajo". Pero al analizarlo por sexos, observamos que coincide con el grupo mujeres, grupo mayoritario, pero no con el grupo de los hombres, cuyo ítem de menor significación es el que hace referencia a la sensación de fatiga al levantarse por la mañana, (Me siento fatigado cuando me levanto por la mañana y tengo que enfrentarme a otro día de trabajo).

Creemos que tales diferencias se pueden explicar desde las consideraciones socioculturales, que tradicionalmente han otorgado a los hombres más fortaleza física, y en el caso de las mujeres una posible fragilidad emocional, que les puede aportar una mayor sensibilidad ante la sensación de frustración (Travers y Cooper, 1997; Abraham, A 1984).

Queremos destacar que como comentamos anteriormente se detecta, que el valor de los porcentajes en el caso de las mujeres es más elevado que en el de los hombres. Todo ello nos lleva a entender que la subescala de Cansancio Emocional tiene una significación más intensa, para el grupo de las mujeres, y por tanto, la elevada puntuación en sus ítems se relacionan más directamente con la manifestación del síndrome de Burnout en el sexo femenino, lo que coincide con aportaciones similadres de otros estudios (Arís, N., 2008; Manasero, M. A., Vázquez, A., Ferrer, V. Fornés, J. Y Fernández, M. C. ,1998). 


\subsection{Subescala Despersonalización}

Tabla 3: Valores obtenidos globalmente y por grupos en DP:

\begin{tabular}{|l|l|l|l|l|} 
COD.ITEM & TOTAL & Hombre & Mujer \\
\hline B05 & $\begin{array}{l}\text { Creo que trato a algunas personas como si } \\
\text { fueran objetos personales }\end{array}$ & $38,24 \%$ & $74,67 \%$ & $24,36 \%$ \\
\hline B10 & $\begin{array}{l}\text { Me he vuelto insensible con la gente desde } \\
\text { que ejerzo esta profesión }\end{array}$ & $34,58 \%$ & $58,86 \%$ & $28,91 \%$ \\
\hline B11 & $\begin{array}{l}\text { Me preocupa el hecho de que este trabajo me } \\
\text { este endureciendo emocionalmente }\end{array}$ & $32,30 \%$ & $59,24 \%$ & $25,07 \%$ \\
\hline B15 & $\begin{array}{l}\text { No me preocupa realmente lo que ocurre a } \\
\text { algunas personas a las que doy servicio }\end{array}$ & $50,34 \%$ & $84,97 \%$ & $30,48 \%$ \\
\hline & $\begin{array}{l}\text { Creo que las personas que trato me culpan de } \\
\text { algunos de sus problemas }\end{array}$ & $42,71 \%$ & $71,93 \%$ & $35,02 \%$ \\
\hline
\end{tabular}

En la subescala Despersonalización, el ítem que más peso tiene en la puntuación total es "No me preocupa realmente lo que ocurre a algunas personas a las que doy servicio".

En esta ocasión y a diferencia de lo sucedido en la anterior subdimensión de Cansancio emocional, no hay una total coincidencia al analizarlo separado por sexos. Es decir, en el caso de los hombres si que el ítem con mayor valor predictivo es el mismo que en la muestra total, y si bien pudiera parecer extraño, puesto que la proporción de hombres es muy menor a la de mujeres, lo cierto es que como hemos visto a lo largo de todo el estudio, la escala de Despersonalización es la de mayor peso en el Burnout en los hombres.

Por último, para el resultado recogido en las mujeres, el ítem de mayor porcentaje es: "Creo que las personas que trato me culpan de algunos de sus problemas". Si analizamos el ítem de menor peso se detectan también, importantes oscilaciones por género.

De manera inversa a lo que sucedía anteriormente, en este caso se observa la coincidencia entre el ítem con menor porcentaje de muestra total, (Me preocupa el hecho de que este trabajo me este endureciendo emocionalmente) y el resultado del grupo de las mujeres.

Para el grupo de los hombres es el de: Me he vuelto insensible con la gente desde que ejerzo esta profesión. Queremos destacar que en esta subescala el valor más elevado de los porcentajes lo aportan los hombres, siendo menos intenso en las mujeres.

Es por lo tanto el caso inverso con la anterior subdimensión, por lo cual entendemos que los ítems de esta subdimensión de despersonalización tienen una mayor relación con la manifestación del Burnout en los hombres. 


\subsection{Subescala Realización personal}

Tabla 4: Valores obtenidos globalmente y por grupos en RP:

\begin{tabular}{|l|l|l|l|l|} 
COD.ITEM & TOTAL & Hombre & Mujer \\
\hline B04 & $\begin{array}{l}\text { Fácilmente comprendo cómo se sienten las } \\
\text { personas }\end{array}$ & $71,53 \%$ & $62,70 \%$ & $70,98 \%$ \\
\hline B07 & $\begin{array}{l}\text { Trato muy eficazmente los problemas de las } \\
\text { personas }\end{array}$ & $74,15 \%$ & $70,61 \%$ & $73,62 \%$ \\
\hline B09 & $\begin{array}{l}\text { Creo que estoy influyendo positivamente } \\
\text { con mi trabajo en la vida de los demás }\end{array}$ & $70,38 \%$ & $67,55 \%$ & $69,52 \%$ \\
\hline B12 & $\begin{array}{l}\text { Me siento muy activo } \\
\text { Bácilmente puedo crear una atmósfera } \\
\text { relajada con las personas a las que doy } \\
\text { servicio }\end{array}$ & $72,63 \%$ & $69,13 \%$ & $71,80 \%$ \\
\hline B17 & $\begin{array}{l}\text { Me siento estimulado después de trabajar } \\
\text { en contacto con personas }\end{array}$ & $71,63 \%$ & $53,66 \%$ & $71,43 \%$ \\
\hline B19 & $\begin{array}{l}\text { He conseguido muchas cosa útiles en mi } \\
\text { profesión }\end{array}$ & $72,29 \%$ & $62,26 \%$ & $74,34 \%$ \\
\hline B21 & $\begin{array}{l}\text { En mi trabajo trato los problemas } \\
\text { emocionales con mucha calma }\end{array}$ & $64,21 \%$ & $72,41 \%$ \\
\hline
\end{tabular}

En este caso, no destacamos las variaciones entre hombres y mujeres en cuanto al ítem de más peso en la subescala, (En mi trabajo trato los problemas emocionales con mucha calma).

Entendemos que hay una total uniformidad en la respuesta puesto que incluso los valores de los porcentajes de cada grupo son muy similares. Ello nos hace atribuirle un valor predictivo elevado. En este sentido, una vez más, es en el ítem de menor valor predictivo donde se recoge una mayor dispersión de las respuestas. Así pues, para la muestra total es el ítem de: Creo que "estoy influyendo positivamente con mi trabajo en la vida de los demás", el cual coincide con el del grupo de las mujeres pero en el caso de los hombres es: Fácilmente puedo crear una atmósfera relajada con las personas a las que doy servicio. Posiblemente dichas diferencias son consecuencia de los diferentes roles atribuidos tradicionalmente a cada sexo.

Queremos destacar que en esta subdimensión no hay diferencias significativas en cuanto a los porcentajes por grupos. Si bien es ligeramente inferior el de los hombres los cierto es que los valores son muy próximos entre todos los grupos.

\section{CONCLUSIONES}

En general podemos concluir que en el estudio de cada dimensión del MBI, se aprecian diferencias significativas por género en cuanto al valor predictivo de 
determinados ítems.

Considerado por cada una de tras tres subescalas cabe decir:

-- En el caso de los ítems de la subescala, Cansancio Emocional, se detecta que en general, el valor de los porcentajes en el caso de las mujeres es más elevado, que en el de los hombres. Ello nos lleva a entender que la subescala de Cansancio Emocional tiene una significación más intensa, para el grupo de las mujeres, y por tanto, la elevada puntuación en sus ítems se relacionan más directamente con la manifestación del síndrome de Burnout en el sexo femenino.

-- La segunda subescala, denominada Despersonalización es donde se evidencia más claramente la diferencia en función del género. Hay evidentes diferencias al analizarlo separado por sexos. Especialmente los resultados obtenidos en los ítems de esta subescala determinan una mayor relación con la manifestación del Burnout en los hombres.

-- La última subescala referente a la Realización personal, no hay diferencias significativas en cuanto a los porcentajes por genero detectándose valores son muy próximos entre todos los grupos.

-- Resulta difícil, a la vista de los pocos estudios centrados en los niveles de educación infantil y educación primaria, poder establecer algún paralelismo de otros estudios, con la información recogida. Pero a la vista de la misma, podemos establecer que la primera de la subescala, Cansancio Emocional está más directamente relacionada con puntuaciones elevadas en el sector femenino. Y la de Despersonalización mayor relación con la manifestación elevada en los hombres.

-- La actitud y un cierto grado de compromiso personal, pueden proporcionar el sustrato para enfrentarse a lo imprevisto, a la búsqueda de respuestas que hay que afrontar y elaborar sobre la marcha en la realidad de cada aula

-- Este estudio pretende establecer un punto de referencia al servició de futuras y necesarias líneas de investigación de la incidencia del Sindrome de Burnout medido por el instrumento MBI en función del genero de los docentes implicados.

Si consideramos la feminización histórica del sector conocer las tendencias en función de tal variable puede permitir un mejor ajuste y rigor en futuros estudios.

Quizás sea precisamente aquí, en el individualismo propugnado por el liberalismo, donde se encuentre el punto de partida erróneo del sistema liberal de medios. Que la comunicación pública deje de estar al servicio del mantenimiento y mejora de la vida social, es decir, que no sirva para compartir objetivos sociales, que no sirva para construir sobre lo que une, manifiesta una contradicción de enorme repercusión. Detrás de ella subyace el debate que sobre el deber ser de la sociedad mantuvo John Dewey contra el liberalismo (Morán, 2009, p. 23). Frente a la compresión de la sociedad como una simple asociación de individuos basada en la cercanía física, lo que él denominaba la "gran sociedad", contraponía la "gran comunidad", una comunidad en la que sus miembros comparten intereses comunes, participando de una unión moral (Dewey 2004, p. 37-138).

Estas observaciones fueron ratificadas por Robert Putnam en su trabajo Bowling alone (2000), la democracia se encontraba en quiebra porque se estaba perdiendo el sentido comunitario y el compromiso cívico, y los medios - en concreto la televisión - eran 
uno de los motivos principales de la situación.

Los medios, ciegos ante las consecuencias de sus acciones, no han sabido ver que dependen totalmente de la viabilidad de la vida pública. Siguiendo a uno de los autores más lúcidos en su crítica al modelo liberal, James Carey, "el periodismo sólo tiene sentido en relación con el público y la vida pública" (1987, p. 14). Como explica Merrit, "una sociedad desinteresada por los asuntos públicos, replegado hacia la vida y asuntos privados, no tiene necesidad ni del periodismo ni de los periodistas" (1995, p. 10).

Las palabras de Lippmann escritas hace más de noventa años (1920, p. 4) resuenan con especial contundencia cuando señalan al periodismo como el causante de la crisis de la democracia. Por ello, hoy, la comunicación pública debe asumir con urgencia la tarea de recuperar su función constructora de la vida social. Para conseguirlo deberá volver a situar en el centro mismo de su razón de ser la idea de interés público y de bien común, quizás sea ésta la única manera de reconstruir el público y, como dice Carey, “devolverlo a la existencia" (1987, p. 4).

\section{BIBLIOGRAFÍA}

Abraham, A (1984). L'enseignant est une persone. Paris, E.S.F. (Traducción al castellano (1986): El enseñante también es una persona. Barcelona: Gedigsa.

Alvarez, M; Obiols, M. (2009) . El proceso de toma de decisones profesionales a traves del coaching. Electronic Journal of Research in Educational Psychology, 7 (2) (n.18), 877900. Obtenido el 22 de mayo de 2009 en

http://www.investigacion-psicopedagogica.org/revista/articulos

Arnau, J (coord.) (1996): Mètodes, dissenys i tècniques en investigación psicològica. Barcelona: Universitat Oberta de Catalunya.

Arís, N. (2009). El sindrome de Burnout en los docentes. Electronic Journal of Research in Educational Psychology, 7 (2) (n.18), 829-848. Obtenido el 22 de mayo de 2009 en http://www.investigacion-psicopedagogica.org/revista/articulos Obtenido el 2 de marzo de 2007 en

http://www.investigacionpcopedagogica.org/revista/articulos/10/espannol/Art 10_134.pdf

Arís, N. (2008). El síndrome de Burnout en los docentes de eduación Infantil y educación primaria en la Zona del Valles Occidental. Barcelona: http://www.tdx.cat/TDX-1027108171248. Fecha consulta 20/03/2010

Álvarez, E. y Fernández, L. (1991a). El síndrome de Burnout o el desgaste profesional (1). Revista Asociación Española de Neuropsiquiatría, 21, 257-265.

Álvarez, E. y Fernández, L. (1991b). El síndrome de Burnout o el desgaste profesional (II). Estudio empírico de los Profesionales Gallegos del área de salud mental. Revisión 
de estudios. Revista Asociación Española de Neuropsiquiatría, 11(39), 257-265.

Calvete, E. y Vila, I (2000). Burnout y síntomas psicológicos: Modelos de medida y relaciones estructurales. Ansiedad y estrés, 6(1), 117-130.

Chermiss, C. (1992). Long-term consequences of Burnout: an exploratory study. En M. Massanero, et al. (1994). Burnout en los profesores: impacto sobre la calidad en la educación, la salud y consecuencias para el desarrollo profesional. (pp.75-85). Memoria final de investigación. Madrid: MEC- CIDE.

Gil-Monte, P. y Peiro, J. M. (1999). Validez Factorial del MASLACH BURNOUT INVENTORY en una muestra multiocupacional. Psicothema, 11(3), (pp679-689).

Gil-Monte, P. y Peiro, J. M. (2000). Un estudio comparativo sobre criterios normativos para el diagnostico del síndrome de quemarse (Burnout). Revista de Psicología del Trabajo y de las Organizaciones, 16(2), 135-149

Manasero, M. A., Vázquez, A., Ferrer, V. Fornés, J. y Fernández, M. C. (1998). Estrés y Burnout en la enseñanza: una aproximación comprensiva. En J. Cerdán y Grañeras Pastrana (eds). Las investigaciones sobre el profesorado (II) 1993-1997, (pp.60-73). Madrid: MEC-CIDE.

Maslach, C. (1982). Burnout: the cost of caring. Englewood Cliffs; N. J. Prentice Hall.

Maslach, C. y Jackson, S. (1981). The measurement of experienced burnout. Journal of Occupational Behavior, 2(99), 99-113.

Seisdedos, N. (1997). Inventario Burnout de Maslach: síndrome del quemado por Estrés Laboral Asistencial. Madrid: TEA ediciones.

Travers y Cooper (1997). El estrés de los profesores. La presión en la actividad docente. Barcelona: Paidós.

\section{Nuria Arís Redó}

Doctora en Ciencias de la Educación por la Universidad Internacional de Cataluña (UIC), con una investigación sobre "El síndrome de Burnout en los maestros". Licenciada en Psicopedagogía y Diplomada en magisterio. Vicedecana de la Facultad de Ciencias de la Educación de la Universidad Internacional de Cataluña. Coordinadora del Máster oficial interdisciplinario de Investigación en CCSS. Acreditación de ANECA de la actividad docente e investigadora. Coautora de los libros "Avances del estudio en la educación emocional", "El estrés laboral de los docente", y otros. 